International Journal of Pure and Applied Mathematics

Volume 115 No. 2 2017, 199-209

ISSN: 1311-8080 (printed version); ISSN: 1314-3395 (on-line version)

url: http://www.ijpam.eu

doi: 10.12732/ijpam.v115i2.1

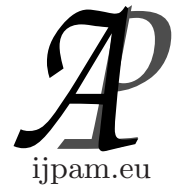

\title{
DYNAMICS OF LESLIE-GOWER PREDATOR-PREY MODEL WITH ADDITIONAL FOOD FOR PREDATORS
}

\author{
Hana Maria Ulfa ${ }^{1}$, Agus Suryanto ${ }^{2}$, Isnani Darti ${ }^{3}$ \\ ${ }^{1,2,3}$ Departement of Mathematics \\ Brawijaya University \\ Jl. Veteran Malang 65145, INDONESIA
}

\begin{abstract}
We consider a Leslie-Gower predator prey model with additional food for predators. Here we investigate the dynamics of the model such as the permanence, determination of equilibrium points and their existence condition as well as their stability properties. It is shown that the model is permanence and has four equilibrium points, i.e., the extinction of both prey and predator point, the extinction of prey point, the extinction of predator and the coexistence point. The point of prey extinction and the coexistence point are conditionally stable while two other equilibrium points are always unstable. It is also shown that the additional food for predators may destabilize the extinction of prey point and at the same time stabilize the coexistence point. Such dynamical behavior agrees with our numerical results.
\end{abstract}

AMS Subject Classification: 34C60, 92D40

Key Words: predator-prey model, Leslie-Gower model, permanence, additional food

\section{Introduction}

The interactions between prey population and predator population in an environment is often modeled by Lotka-Volterra [1]. In its development, Leslie [2] has modified the Lotka-Volterra predator-prey model into Leslie-Gower. The response function in this model is Holling type II, where the predation rate is not only dependent on prey population, but also on the amount of environmental protection. Aziz-Alaoui and Okiye [3] discussed the Leslie-Gower

Received: July 11, 2016

Revised: $\quad$ April 5, 2017

Published: July 14, 2017

$\S_{\text {Correspondence author }}$ (c) 2017 Academic Publications, Ltd. url: www.acadpubl.eu 
predator-prey model as stated below

$$
\begin{aligned}
& \frac{d X}{d t}=(1-X) X-\frac{\delta X Y}{X+m}, \\
& \frac{d Y}{d t}=\beta\left(1-\frac{Y}{X+e}\right) Y,
\end{aligned}
$$

where $X=X(t)$ and $Y=Y(t)$ respectively represent prey and predator populations at time $t$.

The nature of actively moving predators becomes a reason that they can target on more than one prey or switch to other food sources. Considering the limited prey population will require additional food for predators, Srinivasu [4] has studied a Lotka-Volterra predator-prey model with additional food for predators. Later, Sen [5] developed Srinivasu's research [4] in terms of the handling time and the nutritional value of additional food. Many research on Lotka-Volterra predator-prey model with additional food have been conducted [6], [7]. According to Srinivasu [4], the additional food for predator results in a reduced predation target of predator to prey, so it helps the survival of prey population without disturbing predator's growth rate.

In this article, the Leslie-Gower predator-prey model [2] is reconsidered and modified by including the additional food to predators in terms of the handling time and the nutritional value of additional food. The Leslie-Gower predatorprey model with additional food for predators is stated as

$$
\begin{aligned}
& \frac{d X}{d t}=(1-X) X-\frac{\delta X Y}{X+m+n A}, \\
& \frac{d Y}{d t}=\beta\left(1-\frac{Y}{X+e}\right) Y+\frac{\sigma n A Y}{X+m+n A},
\end{aligned}
$$

where $\delta, m, n, A, \beta, e$ and $\sigma$ are positive parameters. As mentioned by Prasad et. al. [6], the term $n A$ represents quantity of additional food perceptible to the predator relative to prey.

\section{Permanence}

In this section we show the permanence of system (1) to ensure that all solutions of this system are bounded. For this purpose, we apply Lemma 1.

Lemma 1. (see Chen, [8]) If $p>0, q>0, \frac{d X}{d t} \geq(p-q X) X$ when $t \geq t_{0}$, and $X\left(t_{0}\right)>0$ then

$$
\lim _{t \rightarrow+\infty} \inf X(t) \geq \frac{p}{q} .
$$


If $p>0, q>0, \frac{d X}{d t} \leq(p-q X) X$ when $t \geq t_{0}$, and $X\left(t_{0}\right)>0$ then

$$
\lim _{t \rightarrow+\infty} \sup X(t) \leq \frac{p}{q}
$$

Proposition 2. Suppose that

$$
\beta(m+n A)^{2}>\delta(\beta(m+n A)+\sigma n A)(1+e),
$$

and let $X(t)$ and $Y(t)$ be any positive solution of system (1), then

$$
A_{1} \leq \lim _{t \rightarrow+\infty} \inf X(t) \leq \lim _{t \rightarrow+\infty} \sup X(t) \leq B_{1}
$$

and

$$
A_{2} \leq \lim _{t \rightarrow+\infty} \inf Y(t) \leq \lim _{t \rightarrow+\infty} \sup Y(t) \leq B_{2}
$$

where

$$
A_{1}=\frac{\beta(m+n A)^{2}-\delta(\beta(m+n A)+\sigma n A)(1+e)}{\beta(m+n A)^{2}},
$$

$A_{2}=e, B_{1}=1$, and

$$
B_{2}=\frac{(\beta(m+n A)+\sigma n A)(1+e)}{\beta(m+n A)} .
$$

Proof. Let's assume that $X(t), Y(t)$ are any positive solution of system (1), then it is obvious that

$$
\begin{aligned}
& \frac{d X(t)}{d t} \leq(1-X(t)) X(t), \\
& \frac{d Y(t)}{d t} \geq \beta\left(1-\frac{Y(t)}{e}\right) Y(t) .
\end{aligned}
$$

From Lemma 1, we have that

$$
\lim _{t \rightarrow+\infty} \sup X(t) \leq B_{1}=1 \text { and } \lim _{t \rightarrow+\infty} \inf Y(t) \geq A_{2}=e .
$$

Hence, for arbitrary $\varepsilon>0$, there exists $T_{1} \geq 0$ such that

$$
X(t) \leq 1+\varepsilon, \text { for } t \geq T_{1}
$$

Substituting (2) into the second equation of system (1) gives

$$
\frac{d Y(t)}{d t} \leq\left(\beta+\frac{\sigma n A}{m+n A}-\frac{\beta Y(t)}{1+\varepsilon+e}\right) Y(t) .
$$


Based on Lemma 1 we get

$$
\lim _{t \rightarrow+\infty} \sup Y(t) \leq \frac{(\beta(m+n A)+\sigma n A)(1+\varepsilon+e)}{\beta(m+n A)} .
$$

If we take $\varepsilon \rightarrow 0$ then we obtain $\lim _{t \rightarrow+\infty} \sup Y(t) \leq B_{2}$. Then, for any $\varepsilon>0$ there exists $T_{2} \geq 0$ such that

$$
Y(t) \leq \frac{(\beta(m+n A)+\sigma n A)(1+e)}{\beta(m+n A)}+\varepsilon, \text { for } t \geq T_{2} .
$$

Furthermore, it is obvious that the first equation of system (1) leads to

$$
\frac{d X(t)}{d t} \geq\left(1-\frac{\delta Y(t)}{m+n A}-X(t)\right) X(t)
$$

Combining (3) and (4) gives

$$
\begin{aligned}
& \quad \frac{d X(t)}{d t} \geq \\
& \left(\frac{\left(\beta(m+n A)^{2}-\delta((\beta(m+n A)+\sigma n A)(1+e)+\varepsilon \beta(m+n A))\right.}{\beta(m+n A)^{2}}-X(t)\right) X(t) .
\end{aligned}
$$

By assuming that

$$
\beta(m+n A)^{2}>\delta(\beta(m+n A)+\sigma n A)(1+e),
$$

and using Lemma 1, we get

$$
\lim _{t \rightarrow+\infty} \inf X(t) \geq \frac{\beta(m+n A)^{2}-\delta((\beta(m+n A)+\sigma n A)(1+e)+\varepsilon \beta(m+n A))}{\beta(m+n A)^{2}} .
$$

Since $\varepsilon$ is arbitrary, we can take $\varepsilon \rightarrow 0$, Thus, $\lim _{t \rightarrow+\infty} \inf X(t) \geq A_{1}$.

By denoting that

$$
\alpha=\min \left\{A_{1}, A_{2}\right\} \text { and } \gamma=\max \left\{B_{1}, B_{2}\right\},
$$

Proposition 2 leads to

$$
\begin{aligned}
& \alpha \leq \min \left\{\lim _{t \rightarrow+\infty} \inf X(t), \lim _{t \rightarrow+\infty}\right.\inf Y(t)\} \\
& \leq \max \left\{\lim _{t \rightarrow+\infty} \sup X(t), \lim _{t \rightarrow+\infty} \sup Y(t)\right\} \leq \gamma
\end{aligned}
$$

This fact shows the permanence of system (1). 


\section{Equilibria and Existence}

An equilibrium point of system (1) is the solution from the following system

$$
\begin{aligned}
& (1-X) X-\frac{\delta X Y}{X+m}=0 \\
& \beta\left(1-\frac{Y}{X+e}\right) Y+\frac{\sigma n A Y}{X+m+n A}=0 .
\end{aligned}
$$

System (1) has four equilibrium points, namely the extinction of both prey and predator point $E_{0}(0,0)$, the extinction of predator point $E_{1}(0, e(1+$ $\left.\frac{\sigma n A}{\beta(m+n A)}\right)$ ), the extinction of prey point $E_{2}(1,0)$ and the survival of both prey and predator point $E_{3}\left(X^{*}, Y^{*}\right)$, with $Y^{*}=\frac{-\left(X^{*}\right)^{2}+(1-m-n A) X^{*}+m+n A}{\delta}$ must be positive and $X^{*}$ is all possible positive roots of cubic equation

$$
\left(X^{*}\right)^{3}+3 v_{1}\left(X^{*}\right)^{2}+3 v_{2} X^{*}+v_{3}=0,
$$

where

$$
\begin{aligned}
& v_{1}=\frac{\delta \beta+2 \beta m+2 \beta n A-\beta}{3 \beta}, \\
& v_{2}=\frac{\delta \beta m+\delta \beta n A+\delta \beta e-2 \beta m+\beta m^{2}+2 \beta m n A-2 n A \beta+\beta(n A)^{2}+\delta \sigma n A}{3 \beta}, \\
& v_{3}=\frac{\delta \beta e m+\delta \beta e n A-\beta m^{2}-2 \beta m n A-\beta(n A)^{2}+\delta \sigma n A e}{\beta} .
\end{aligned}
$$

Using the following transformation

$$
s=X^{*}+v_{1},
$$

equation (5) can be written as

$$
f(s)=s^{3}+3 g s+h,
$$

where $g=v_{2}-v_{1}^{2}$ and $h=v_{3}-3 v_{1} v_{2}+2 v_{1}^{3}$. All possible positive roots of equation (6) can be evaluated by Cardan's method as stated in Lemma 3.

Lemma 3. Cai et. al. [9]. The existence of positive root of equation (6) can be described as follows.

1. If $h<0$ then equation (6) has a unique positive root. 
2. If $h>0$ and $g<0$ :

(a) if $h^{2}+4 g^{3}=0$, then equation (6) has a unique positive root of multiplicity two,

(b) if $h^{2}+4 g^{3}<0$, then equation (6) has two distinct positive roots.

3. If $h=0$ and $g<0$, then equation (6) has a unique positive root.

If equation (6) has two positive roots, then the roots are

$$
s_{1}=\frac{\sqrt[3]{\left(-h+4 \sqrt{4 g^{3}+h^{2}}\right)^{2}}-4 g}{2 \sqrt[3]{\left(-h+4 \sqrt{4 g^{3}+h^{2}}\right)^{2}}} \text { and } s_{2}=-\frac{s_{1}}{2}+\frac{\sqrt{s_{1}^{3}+4 h}}{2 \sqrt{s_{1}}}
$$

respectively. Meanwhile, if equation (6) has only one positive root, then the root is $s_{1}=\frac{\sqrt[3]{\left(-h+4 \sqrt{4 g^{3}+h^{2}}\right)^{2}}-4 g}{2 \sqrt[3]{\left(-h+4 \sqrt{4 g^{3}+h^{2}}\right)^{2}}}$.

\section{Stability of the Equilibria}

The local stability of each equilibrium point of system (1) is shown in Teorema 4.

Theorem 4. Stability of Equilibria

1. Equilibrium point $E_{0}=(0,0)$ is unstable node.

2. Equilibrium point $E_{1}=\left(0, e\left(1+\frac{\sigma n A}{\beta(m+n A)}\right)\right)$ is locally stable if

$$
\frac{\beta(m+n A)^{2}}{\delta(\beta(m+n A)+\sigma n A)}<e .
$$

3. Equilibrium point $E_{2}=(1,0)$ is a saddle point.

4. $E_{3}=\left(X^{*}, \frac{-\left(X^{*}\right)^{2}+(1-m-n A) X^{*}+m+n A}{\delta}\right)$ is locally stable if

$$
\left(X^{*}+\beta\right)\left(X^{*}+m+n A\right)+\sigma n A>\left(1-X^{*}\right) X^{*},
$$

and

$$
\omega>\frac{\delta \sigma n A}{\left(X^{*}+m+n A\right)^{2}}
$$

where $\omega=\frac{\beta\left(X^{*}+e\right)\left(X^{*}+m+n A\right)+\beta\left(1-X^{*}\right)(m+n A-e)}{\left(X^{*}+e\right)^{2}}$. 
Proof. 1. The Jacobian matrix of system (1) at $E_{0}$ is

$$
J\left(E_{0}\right)=\left[\begin{array}{cc}
1 & 0 \\
0 & \beta+\frac{\sigma n A}{m+n A}
\end{array}\right] .
$$

Both eigenvalues of $J\left(E_{0}\right)$ are positive, therefore $E_{0}$ is unstable node.

2. The Jacobian matrix of system $(1)$ at $E_{1}$ is $J\left(E_{1}\right)=\left[\begin{array}{cc}a_{11} & 0 \\ a_{21} & a_{22}\end{array}\right]$ where

$$
\begin{gathered}
a_{11}=1-\frac{\delta e\left(1+\frac{\sigma n A}{\beta(m+n A)}\right)}{m+n A}, \\
a_{21}=\frac{\beta\left(e\left(1+\frac{\sigma n A}{\beta(m+n A)}\right)\right)^{2}}{e^{2}}-\frac{\sigma n A e\left(1+\frac{\sigma n A}{\beta(m+n A}\right)}{(m+n A)^{2}}, \\
a_{22}=\frac{-\beta(m+n A)-\sigma n A}{m+n A} .
\end{gathered}
$$

The eigenvalues of $J\left(E_{1}\right)$ are

$$
\lambda_{1}=1-\frac{\delta e\left(1+\frac{\sigma n A}{\beta(m+n A)}\right)}{m+n A} \text { and } \lambda_{2}=\frac{-\beta(m+n A)-\sigma n A}{m+n A}<0 .
$$

Thus, $E_{1}$ is stable if $\frac{\beta(m+n A)^{2}}{\delta(\beta(m+n A)+\sigma n A)}<e$.

3. At $E_{2}$, the Jacobian matrix of system $(1)$ is $J\left(E_{2}\right)=\left[\begin{array}{cc}-1 & -\frac{\delta}{1+m+n A} \\ 0 & \beta+\frac{\sigma n A}{1+m+n A}\end{array}\right]$. Clearly that one of eigenvalues of $J\left(E_{2}\right)$ is positive, so that $E_{2}$ is a saddle point.

4. The Jacobian matrix at $E_{3}$ is

$$
J\left(E_{3}\right)=\left[\begin{array}{cc}
-X^{*}+\frac{\delta X^{*} Y^{*}}{\left(X^{*}+m+n A\right)^{2}} & -\frac{\delta X^{*}}{X^{*}+m+n A} \\
\frac{\beta\left(X^{*}\right)^{2}}{\left(X^{*}+e\right)^{2}}-\frac{\sigma n A Y^{*}}{\left(X^{*}+m+n A\right)^{2}} & -\frac{\beta Y^{*}}{X^{*}+e}
\end{array}\right] .
$$

The trace and determinant of $J\left(E_{3}\right)$ are respectively given by

$$
\operatorname{Trace}\left(J\left(E_{3}\right)\right)=\frac{\left(1-X^{*}\right) X^{*}-\left(X^{*}+\beta\right)\left(X^{*}+m+n A\right)-\sigma n A}{X^{*}+m+n A}
$$


and

$$
\operatorname{Det}\left(J\left(E_{3}\right)\right)=\omega-\frac{\delta \sigma n A}{\left(X^{*}+m+n A\right)^{2}} .
$$

$E_{3}$ is stable if $\operatorname{Trace}\left(J\left(E_{3}\right)\right)<0$ and $\operatorname{Det}\left(J\left(E_{3}\right)\right)>0$. These conditions are equivalent to $(8)$ and $(9)$.

\section{Numerical Simulations}

To illustrate our analytical results, we show some numerical simulations using different parameter values.

Numerical simulation 1. First, we perform numerical simulation by taking $\delta=0.7, m=0.7, A=6, \beta=0.45, e=0.8,=3, n=0.3$. It is found that $h<0$, hence equation (6) has one positive $\operatorname{root} s^{*}>0$. Equilibrium point $E_{3}$ does not exist because $s^{*}<v_{1}$. Therefore, there exist only three equilibrium points namely $E_{0}(0,0), E_{1}(0,4.64)$ and $E_{2}(1,0)$. Based on the calculation, it is noted that the parameter values satisfy condition (7). Thus, according to Theorema $4, E_{1}$ is stable. This behavior is clearly seen from the phase portrait shown in Figure 1where all solutions with various initial values are convergent to $E_{1}$.

Numerical simulation 2. We observe from conditions (7-9) that changing the coefficient of additional food ( $n$ or $A$ ) may change the stability properties of $E_{1}$ and $E_{3}$. Indeed, increasing the value of $n$ or $A$ will also increase the value of left hand side of condition (7); meaning that it also increases the possibility of $E_{1}$ to be unstable. On the other hand, increasing the value of $n$ or $A$ will certainly increase the value of left hand side of conditions (8) and (9), and will simultaneously decrease the value of right hand side of condition (9). Hence, it increases the possibility of $E_{3}$ to be stable. To see this behavior, we perform a simulation using the same parameter values as in Simulation 1, except for $n=0.8$. Notice that we have increase the coefficient of additional food for predator. These parameter values produce $h<0$, meaning that equation (6) has unique $s^{*}>0$. Since $s^{*}>v_{1}$, system (1) has four equilibrium points, namely $E_{0}(0,0), E_{1}(0,5.4545), E_{2}(1,0)$, and $E_{3}(0.1921,6.5692)$. It is found that by changing the value of $n=0.3$ into $n=0.8$, condition (7) becomes unsatisfied, while conditions (8) and (9) are fulfilled. Thus, $E_{1}$ becomes unstable and $E_{3}$ is now stable. This behavior is obviously seen in Figure 2, where all solutions with various initial values converge to $E_{3}$. 


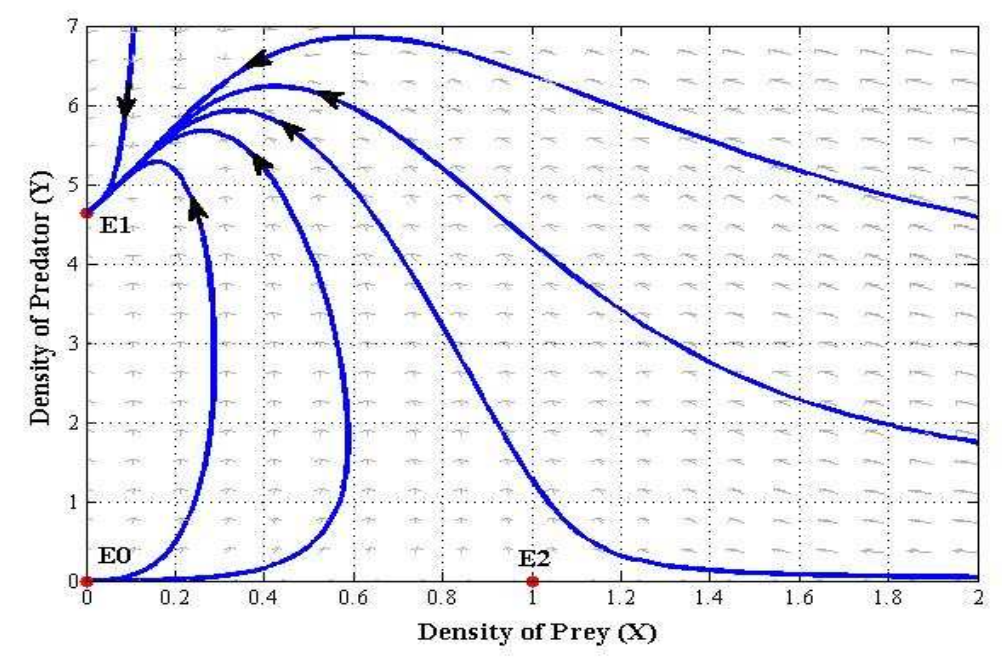

Figure 1: The portrait of model (1) with $\delta=0.7, m=0.7, A=6, \beta=$ $0.45, e=0.8,=3, n=0.3$

\section{Conclusions}

We have shown that the Leslie-Gower predator-prey model with additional food for predator is a permanence system, indicating that the solution of system is bounded. It is also found that the model has four equilibrium points, namely the extinction of both populations point $\left(E_{0}\right)$, the extinction of predator point $\left(E_{1}\right)$, the extinction of prey point $\left(E_{2}\right)$, and both populations are able to survive $\left(E_{3}\right)$. $E_{0}$ and $E_{2}$ are always unstable, whereas $E_{1}$ and $E_{3}$ are stable under certain conditions. Increasing the coefficient of additional food for predator $(n)$ may stabilize equilibrium $E_{3}$ and at the same time destabilize equilibrium $E_{1}$.

\section{Acknowledgments}

This work was supported by the Directorate of Research and Community Service, The Directorate General of Strengthening Research and Development, the Ministry of Research, Technology and Higher Education (Brawijaya University); Indonesia: Contract Number: 137/SP2H/LT/DRPM/III/2016 dated March 


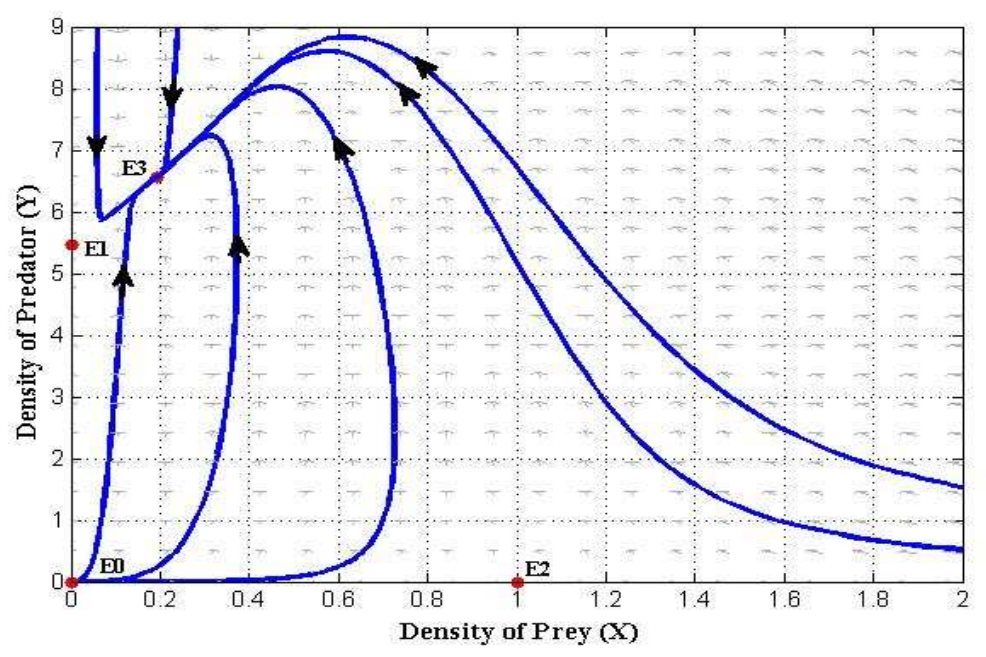

Figure 2: The portrait of model (1) with $\delta=0.7, m=0.7, A=6, \beta=$ $0.45, e=0.8,=3, n=0.8$

10,2016 .

\section{References}

[1] N. Finizio, G. Ladas, An Introduction to Differential Equations with Difference Equation, Fourier Series, and Partial Differential Equations, Wadsworth Publishing Company, Belmont, California (1982).

[2] P. H. Leslie, Some Further Notes on the Use of Matrices in Population Mathematics, Biometrika 35 (1948), 213-245.

[3] M. A. Aziz-Alaoui, M. D. Okiye, Boundedness and Global Stability for a Predator-Prey Model with Modified Leslie-Gower and Holling-type II schemes, Applied Mathemathics Letters 16 (2003), 1069-1075.

[4] P. D. N. Srinivasu, B. S. R. V. Prasad, M. Venkatesulu, Biological Control Through Provision of Additional Food to Predators, Theoretical Population Biology, University of Missouri, Kansas City, USA (2007), 111-120.

[5] M. Sen, P. D. N. Srinivasu, M. Banerjee, Global Dynamics of an Additional Food Provided Predator-Prey System with Constant Harvest in Predators, Applied Mathematics and Computation (2015), 193-211.

[6] B. S. R. V. Prasad, M. Banerjee, P. D. N. Srinivasu, Dynamics of Additional Food Provided Predator-Prey System with Mutually Interfering Predators, Mathematical Biosciences (2013), 176-190. 
[7] B. Sahoo, S. Poria, Effects of Additional Food in a Delayed Predator-Prey Model, Mathematical Biosciences (2015), 62-73.

[8] F. D. Chen, On a Nonlinear Non-Autonomous Predator-Prey Model with Diffusion and Distributed Delay, Journal of Computational and Applied Mathematics 180, No. 1 (2005), 33-49.

[9] Y. Cai, C. Zhao, Wang W., J. Wang, Dynamics of Leslie-Gower Predator- Prey Model With Additive Allee Effect, Applied Mathematical Modelling 39 (2005), 2092-2106. 
